\title{
Assessment of Myocardial Function from Cine Cardiac MRI Using a Novel 4D Tracking Approach
}

\author{
Hisham Sliman ${ }^{1,2}$, Ahmed Elnakib ${ }^{1,3}$, Garth M Beache ${ }^{4}$, Adel Elmaghraby ${ }^{2}$ and Ayman El-Baz ${ }^{1 *}$ \\ ${ }^{1}$ Bioimaging Laboratory, Bioengineering Department, University of Louisville, Louisville, USA \\ ${ }^{2}$ Computer Engineering and Computer Science Department, University of Louisville, Louisville, USA \\ ${ }^{3}$ Electronics and Communications Engineering, Mansoura University, Mansoura, Egypt \\ ${ }^{4}$ Department of Radiology, University of Louisville, Louisville, USA
}

\begin{abstract}
A novel approach for assessing the myocardial function using 4D cine cardiac magnetic resonance imaging (CMRI) is proposed. The myocardial function is assessed by estimating the left ventricle (LV) wall thickening based on a novel 4D tracking approach that tracks the LV wall during the cardiac cycle. Initially, the proposed 4D approach tracks the surface points on the LV wall by solving a 3D Laplace equation between each two subsequent LV surfaces. In order to remove inconsistencies and preserve the anatomy of the heart wall during the tracking process, the initial locations of the tracked LV surface points are iteratively adjusted through an energy minimization cost function using a generalized Gauss-Markov random field (GGMRF) image model. Using the iteratively adjusted solution of the 3D Laplace equation, the myocardial wall thickening is estimated by co-allocation of the corresponding points, or matches between the endocardium and epicardium surfaces of the LV wall. Experimental results on in vivo data demonstrate that our approach outperforms 2D wall thickening estimation approaches.
\end{abstract}

Keywords: Strain; Cine; Cardiac; MRI; Laplace; Tracking; Left ventricle

\section{Introduction}

Assessment of the cardiac performance is clinically important for diagnosing and managing patients with cardiovascular diseases [1-4]. To quantify the cardiac performance, cardiac studies have employed a number of important indicators, such as the wall thickness, wall thickening, and functional strain [5-8]. These indicators are traditionally estimated using a number of different medical image modalities. For example, tagged MRI or ultrasound are the traditional medical image modalities for estimating the functional strain, whereas cine CMRI is the traditional technique for estimating wall thickening. The ultimate goal of this work is to develop a comprehensive framework for estimating different performance indexes of the heart (e.g., functional strain and wall thickening) from the same image modality (i.e., cine CMRI) in a way that avoids the inconsistency between different estimated indexes. This inconsistency results from the inter-slice variability and different image resolutions of the different image modalities. In this work, we will focus on estimating the wall thickening from cine CMRI using a novel $4 \mathrm{D}$ approaches for tracking the heart wall over the cardiac cycle. Next, we will overview the current methods for estimating the wall thickening as well as their limitations.

\section{Related work on wall thickness analysis}

Wall thickening is an important indicator for myocardium dysfunction, which is more accurate than wall motion analysis [912]. It is typically assessed by visual inspection, which is preferred clinically for practical purposes [13]. However, this is obviously a time consuming process and is prone to considerable intra- and interobserver variability which is a drawback $[9,14-16]$. To overcome this, local myocardial wall thickness is derived, automatically or semiautomatically, after tracing the endocardial and epicardial boundaries in all short-axis images. Prasad et al. [17] proposed to measure the myocardial thickening in CMRI more reliably by solving a partial differential Laplace equation. However, to reduce the effects of segmentation errors in the wall thickness estimation, a further step of manual adjustment was performed by a clinical expert. Recently,
Khalifa et al. [18] proposed an automated framework for analyzing the wall thickness and thickening function by solving the 2D Laplace equation. However, their method is based on $2 \mathrm{D}$ analysis and did not take into account the 3D motion of the cardiac wall (i.e., out-of-plane motion). Therefore, there is a need for developing more methods for more accurate wall thickness analysis.

\section{Limitations of existing works and our approach}

In summary, the abovementioned frameworks for analyzing the regional function (i.e., wall thickening) are not sufficiently accurate and reliable for several reasons: $(i)$ visual inspection is obviously a time consuming process and is prone to considerable intra- and interobserver variability, and (ii) current $2 \mathrm{D}$ methods for wall thickening estimation lead to inaccurate measurements because they do not take into account the 3D motion of the heart (i.e., out-of-plane motion).

To overcome the aforementioned limitations, we propose a novel PDE-based method to estimate the wall thickening from 4D cine CMRI based on tracking the LV wall geometry. To achieve this goal, we develop a 4D (3D+time) approach to track the $\mathrm{LV}$ wall points based on solving the 3D Laplace equation between each two successive surfaces over the cardiac cycle. To preserve the anatomy of the heart wall, the initially tracked surface points are iteratively refined through an energy minimization cost function using a generalized Gauss-Markov random field (GGMRF) image model. Our ultimate goal is to use the same image modality (i.e., cine CMRI) to estimate performance indexes of the heart (e.g., wall thickening and functional strains) in order to

*Corresponding author: Ayman El-Baz, Bioimaging Laboratory, Bioengineering Department, University of Louisville, Louisville, KY 40292, USA, Tel: 502-8525092; Fax: 502-852-6806; E-mail: aselba01@louisville.edu

Received June 03, 2014; Accepted June 25, 2014; Published June 27, 2014

Citation: Sliman H, Elnakib A, Beache GM, Elmaghraby A, El-Baz A (2014) Assessment of Myocardial Function from Cine Cardiac MRI Using a Novel 4D Tracking Approach. J Comput Sci Syst Biol 7: 169-173. doi:10.4172/jcsb.1000152

Copyright: $\odot 2014$ Sliman H, et al. This is an open-access article distributed under the terms of the Creative Commons Attribution License, which permits unrestricted use, distribution, and reproduction in any medium, provided the original author and source are credited. 
obtain more correlated and accurate indexes, which have the ability to quantify meaningful effects in treatment and physiological studies.

\section{Methods}

The proposed framework for estimating the wall thickening of the heart from cine CMRI is schematized in Figure 1. The segmentation of the LV wall borders can be obtained using any segmentation technique, e.g., using the method in [18] (more about segmentation methods can be found in [19]). In this paper, we focus on the tracking of the LV wall points and the assessment of the myocardial function. Details of the proposed framework are described below.

\section{D tracking of the LV wall points}

Initial tracking using the solution of the 3D Laplace equation: In order to estimate the heart performance indexes, the surface points of the myocardium should be tracked over the cardiac cycle. In this work, we propose a geometrically motivated approach to track the surface points on the LV wall through the cardiac cycle. Our method tracks the LV surfaces' points by solving the Laplace equation between each two successive surfaces [20-23] (we denote one as the reference surface and the other one as the target surface). The Laplace equation is a secondorder linear PDE, which takes the form:

$$
\nabla^{2} \gamma=\frac{\partial^{2} \gamma}{\partial x^{2}}+\frac{\partial^{2} \gamma}{\partial y^{2}}+\frac{\partial^{2} \gamma}{\partial z^{2}}=0
$$

where $\gamma(x, y, z)$ is the estimated electric field between the surfaces. The solution $\gamma$ between two surfaces results in intermediate equipotential surfaces and streamlines (field lines), being everywhere orthogonal to all equipotential surfaces and establishing natural voxel-to-voxel correspondences between the surfaces. In order to estimate $\gamma(x, y, z)$ , we used a second order central differences method and the iterative Jacobi approach:

$$
\begin{aligned}
\gamma^{i+1}(x, y, z)=\frac{1}{6}\{ & \gamma^{i}\left(x+\theta_{x}, y, z\right)+\gamma^{i}\left(x-\theta_{x}, y, z\right)+\gamma^{i}\left(x, y+\theta_{y}, z\right) \\
& \left.+\gamma^{i}\left(x, y-\theta_{y}, z\right)+\gamma^{i}\left(x, y, z+\theta_{z}\right)+\gamma^{i}\left(x, y, z-\theta_{z}\right)\right\}
\end{aligned}
$$

where $\gamma^{i}(x, y, z)$ is the estimated electric field at $(x, y, z)$ during the $i^{\text {th }}$ iteration; and $\theta_{x}, \theta_{y}, \theta_{z}$ are the step length or resolution in $x, y, z$ directions, respectively. Basic steps of the proposed Laplace-based tracking methodology are summarized in Algorithm 1.

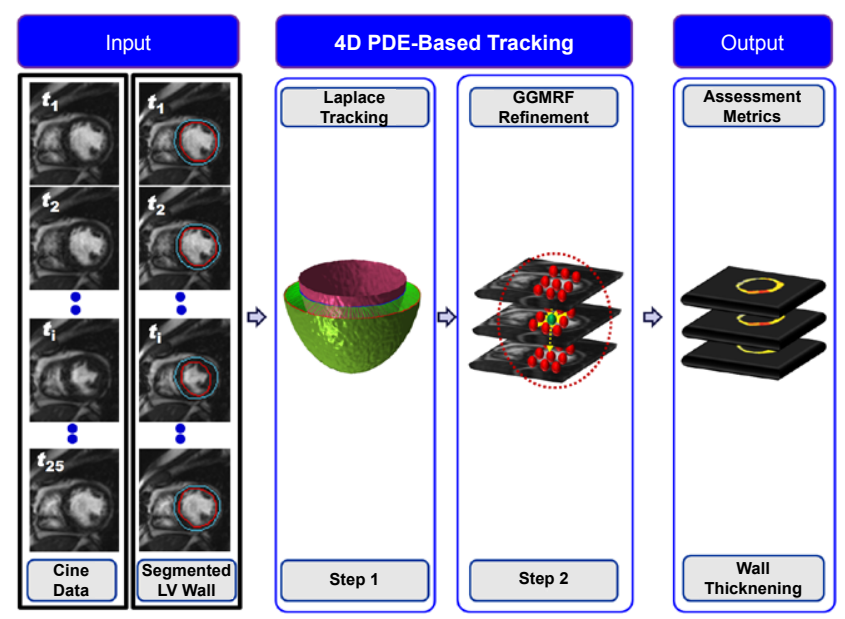

Figure 1: The proposed framework for estimating the performance indexes of the heart using cine CMRI.

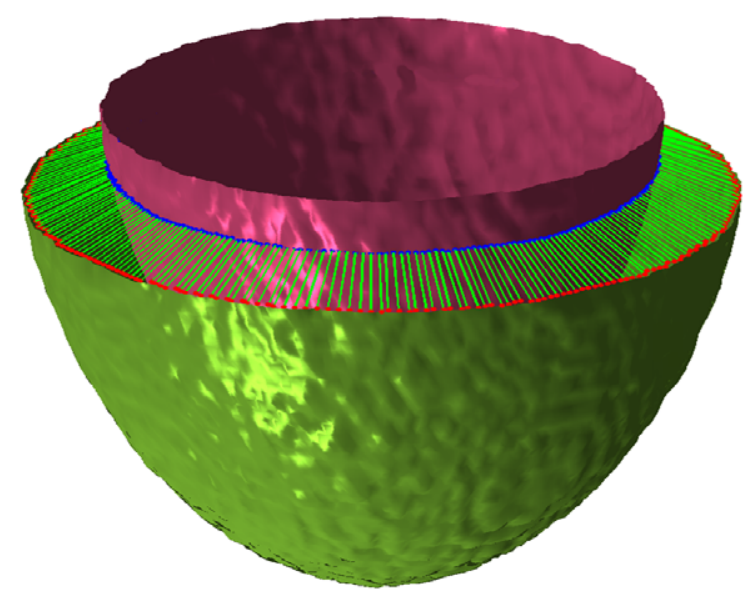

Figure 2: Schematic illustration of the 2-nearest voxels.

\footnotetext{
Algorithm 1 Solution of the 3D Laplace Equation Between Two Surfaces

1) Find the $3 \mathrm{D}$ edges of both $\mathrm{LV}$ wall surfaces.

Initial condition: Set the maximum and minimum potential $\gamma$ at the target and reference surfaces, respectively.

Estimate $\gamma$ between both surfaces using Eq. (2).

Iterate Step 3 until convergence is achieved (i.e., there is no change in estimated $\gamma$ values between iterations).
}

GGMRF-based refinement: In order to avoid any anatomical distortions that result from solving the Laplace equation, we employ a smoothness constraint to preserve the LV wall anatomy. The introduced constraint preserves the relative position between the neighboring voxels on the target LV wall surface through iterative energy minimization using a GGMRF image model [24] on the initially tracked points. Each tracked point on the target is iteratively refined by a GGMRF image model [13] using the voxels neighborhood system (N-nearest neighbors) (Figure 2).

Given the $N$-nearest neighbors of each point on the target surface, the location of each point is refined using its maximum A posteriori (MAP) estimates and voxel-wise stochastic relaxation (iterative conditional mode (ICM) [25]) that jointly optimize $x, y$ and $z$ spatial coordinates using $[18,26]$ :

$$
\begin{gathered}
\tilde{\mathbf{p}}_{\mathrm{s}}=\underset{\tilde{\mathbf{p}}_{s}=\left(\tilde{x}_{s}, \tilde{y}_{,}, z_{s}\right)}{\arg \min }\left\{\left|x_{s}-\tilde{x}_{s}\right|^{\alpha}+\rho^{\alpha} \lambda^{\beta} \sum_{r \in N} \eta_{s, r}\left|\tilde{x}_{s}-x_{r}\right|^{\beta}+\left|y_{s}-\tilde{y}_{s}\right|^{\alpha}+\rho^{\alpha} \lambda^{\beta} \sum_{r \in N} \eta_{s, r}\left|\tilde{y}_{s}-y_{r}\right|^{\beta}\right. \\
\left.+\left|z_{s}-\tilde{z}_{s}\right|^{\alpha}+\rho^{\alpha} \lambda^{\beta} \sum_{r \in N} \eta_{s, r}\left|\tilde{z}_{s}-z_{r}\right|^{\beta}\right\}
\end{gathered}
$$

where $\mathbf{p}_{\mathrm{s}}=\left(x_{s}, y_{s}, z_{s}\right)$ and $\tilde{\mathbf{p}}_{\mathrm{s}}=\left(\tilde{x}_{s}, \tilde{y}_{s}, \tilde{z}_{s}\right)$ denote the initial tracked points' locations and their expected estimates; $N$ is the number of nearest neighbors (Figure 2); $\eta_{s, r}$ is the GGMRF potential, and $\rho$ and $\lambda$ are scaling factors. In addition to $N$, the parameter $\beta \in[1.01,2.0]$ controls the refinement level (e.g., $\beta=2$ for smooth vs. $\beta=1.01$ for relatively abrupt edges). The parameter $\alpha \in\{1,2\}$ determines the Gaussian, $\alpha=2$, or Laplace, $\alpha=1$, prior distribution of the estimator. Our experiments below were conducted with $\rho=1, \lambda=5, \beta=1.01, \alpha=2$ and $\eta_{s, r}=\sqrt{2}$ for all directions. 


\section{Wall thickening assessment}

The changes in the wall thickness during systole of the cardiac cycle (i.e., the wall thickening) are obtained by accurate co-allocation of the corresponding points, or matches, between the inner and outer surfaces of the LV wall by solving the 3D Laplace equation. The wall thickness and thickening are estimated using the geodesic distances between the estimated corresponding point pairs. A typical example of finding the point-wise correspondences between the inner and outer surfaces of the heart wall using the solution of the 3D Laplace equation is shown in Figure 3.

\section{Results and Discussion}

The proposed framework has been tested on 15 independent cine CMR datasets obtained from six patients, with prior myocardial infarctions that were documented by both clinical indexes and viability MRI [22]. Short-axis images were obtained using a $1.5 \mathrm{~T}$ Espree system, Siemens Medical Solutions, USA Inc., with phased array wrap-around reception coils. Breath-hold cine imaging was done using balanced steady-state free precession. Typical parameters were: TR: $4.16 \mathrm{~ms}$; TE: $1.5 \mathrm{~ms}$; flip angle: $80^{\circ}$; average k-space lines per segment: 12 ; inplane resolution: $1.4 \times 3.1 \mathrm{~mm}^{2}$; and slice thickness: $8 \mathrm{~mm} .25$ temporal image frames were obtained for each slice. The patients were part of an institutional review board (IRB)-approved study investigating a novel myoregeneration therapy, and all patients had given informed consent before imaging.

\section{In-Vivo validation}

In order to validate our method, we compare the estimated values of the wall thickening to the ground truth (GT) values, which were estimated using a set of landmark points that were selected and traced manually by a radiologist throughout the cardiac cycle. Comparison results between our method and the GT for estimating wall thickening are presented. As demonstrated, our estimation is close to the GT as documented by the statistical paired t-test [27] with $P$-value greater than 0.05 , which indicates non-significant difference.

To highlight the advantage of the proposed 3D method for estimating the wall thickening, we compare our method with the $2 \mathrm{D}$ method proposed by Khalifa et al. [18]. Unlike our method, the 2D analysis [18] shows a significant difference from the GT (the paired $\mathrm{t}$-test $\mathrm{P}$-value is less than 0.05). This is due to the fact that $2 \mathrm{D}$ methods do not take into account the 3D heart motion and out-of-plane motion that lead to inaccurate estimation of the wall thickening metric [28]. So our method can provide more accurate results, which make it more suitable for clinical applications [29-32]. These results highlight the advantages of the proposed framework.

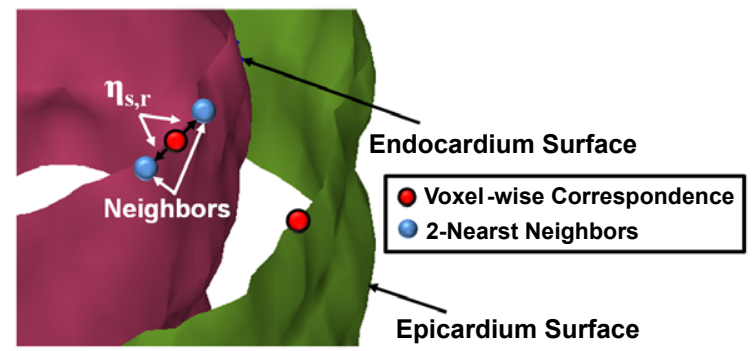

Figure 3: Coallocation of corresponding LV wall points for a patient data using the proposed method. The inner and outer LV wall surfaces are shown in pink and green respectively.

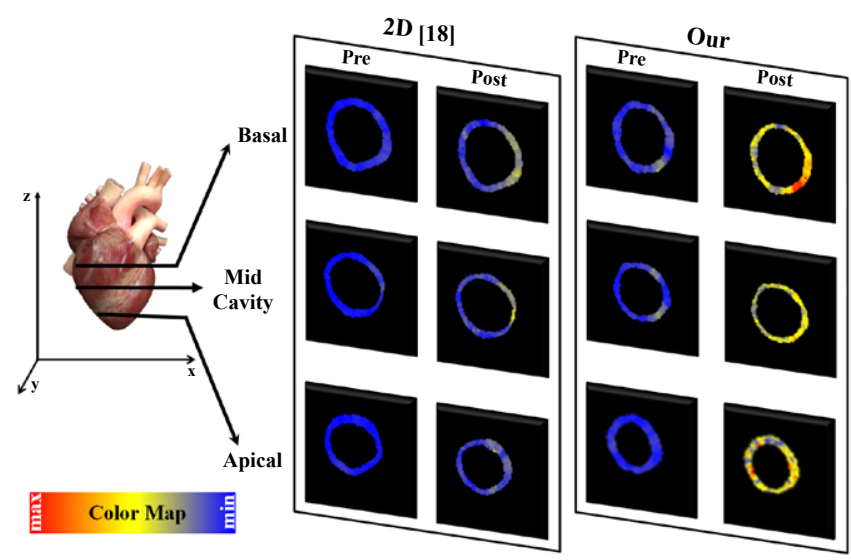

Figure 4: Pre- and post-thickening analysis using the 2D method proposed in [18] and our proposed method for a patient enrolled in this study. The results are projected on 2D basal, mid-cavity, and apical cross-sections for illustration.

\section{Clinical applications}

Wall thickness analysis has been used in many clinical applications. These applications include the detection, quantification, and follow up the treatment of different cardiac diseases, such as hypertrophic cardiomyopathy [29], hypertensive heart disease [30], amyloid heart disease [31], and chronic ischemic heart disease [32]. To emphasize the potential of using the wall thickening to document changes with treatment, we have tested our method in research participants with chronic ischemic heart disease and heart damage who underwent a stem-cell myogeneration therapy. For visual assessment of the wall thickening $(\Delta)$ functional parameter, we use a voxel-wise parametric (color-coded) map. To derive these functional maps, each $\Delta$ value is normalized by relating it to the maximum value measured in the whole volume for the pre- or post-treatments, for the given subject. Figures 4 and 5 present the parametric maps for the $\Delta$ values over multiple crosssections for pre- and post-therapy of four subjects. As shown in Figure 4 , our $4 \mathrm{D}$ method can better detect the variability of the wall thickening than the $2 \mathrm{D}$ method proposed by Khalifa et al. [18]. These results emphasize the potential of using the wall thickening to document changes with treatment that were consistent with improvements in patient condition, as documented by clinical indexes. This lends encouragement for the proposed framework to detect and quantify meaningful effects in treatment and physiological studies.

\section{Future work}

The proposed framework has been preliminary tested on six patients over multiple time sessions. In order to further test and quantify the accuracy of the proposed approach, we plan to acquire more datasets with different heart abnormalities and test the accuracy of our approach with respect to ground truth landmark points, selected by a radiologist. In addition, to avoid the human variability, we plan to test our approach using synthetic simulated phantoms with known ground truth wall thicknesses and different noise levels to further quantify the accuracy and robustness of the method. Furthermore, our future work include the estimation of other global (e.g., ejection fraction) and local (e.g., functional strain) assessment metrics, derived from cine CMRI, for patients enrolled in our case study, stem-cell myoregeneration therapy. 

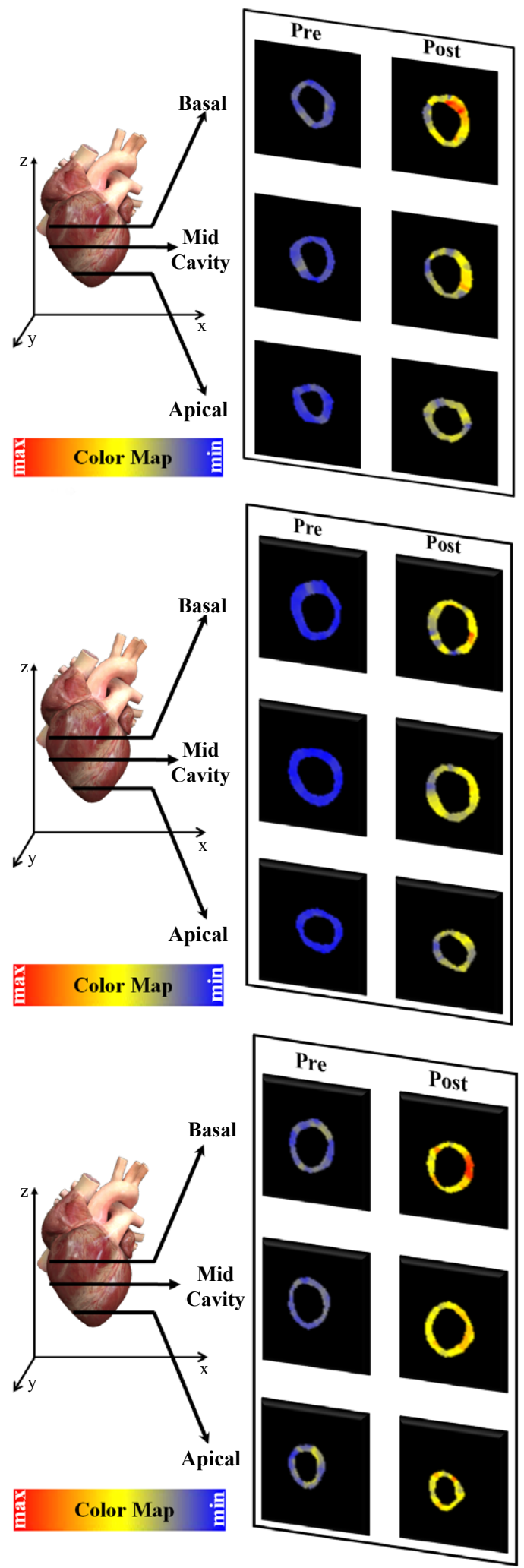

Figure 5: Pre- and post-thickening analysis for other three subjects ((a), (b) and (c)) enrolled in this study, projected on 2D basal, mid-cavity, and apical cross-sections for illustration.

\section{Conclusion}

A novel 4D (3D+time) tracking approach for accurate assessment of myocardium function using cine CMRI is presented. The experimental results on in vivo data demonstrated the ability of the proposed approach for detecting the out-of plane heart motion which leads to accurate estimation of $4 \mathrm{D}$ wall thickening assessment metric. The main benefit of $4 \mathrm{D}$ regional analysis using cine CMRI is that it allows better detection of the variability of the wall thickening than the 2D methods. The results presented in this study emphasize the potential of using the proposed $4 \mathrm{D}$ wall thickening analysis to document changes with treatment and to detect and quantify meaningful effects in physiological studies.

\section{References}

1. Henein MY (2010) Heart failure in clinical practice. Cardiology \& Angiology $1-320$.

2. Nitzken M, Beache G, Elnakib A, Khalifa F, Gimel'farb G, et al. (2012) Improving full-cardiac cycle strain estimation from tagged CMR by accurate modeling of $3 D$ image appearance characteristics. Proceeding IEEE International Symposium on Biomedical Imaging 462-465.

3. Sliman H, Khalifa F, Elnakib A, Soliman A, Beache GM, et al. (2013) A new segmentation based tracking framework for extracting the left ventricle cavity from cine cardiac MRI. Proceeding 685-689.

4. Sliman H, Khalifa F, Elnakib A, Soliman A, El-Baz, et al. (2013) Myocardial borders segmentation from cine MR images using bidirectional coupled parametric deformable models. Med Phys40:092302.

5. Beache GM, Wedeen V, Dinsmore R, (1993) Magnetic resonance imaging evaluation of left ventricular dimensions and function and pericardial and myocardial disease. Coron Artery Dis 4: 328-333.

6. Nitzken M, Beache G, Elnakib A, Khalifa F, Gimel'farb G, et al. (2012) Accurate modeling of tagged CMR 3D image appearance characteristics to improve cardiac cycle strain estimation. Proceeding IEEE International Conference on Image Processing 521-524.

7. Elnakib, Beache GM, Sliman H, Gimel'farb G, Inanc T, et al. (2013) A nove laplace-based method to estimate the strain from cine cardiac magnetic resonance images. Proceeding IEEE Intenational Conference Image Processing 690-694.

8. Elnakib, Beache GM, Gimel'farb G, Inanc T, El-Baz A (2013) Validating a new methodology for strain estimation from cardiac cine MRI. Proceeding International Symposia Computational Models for Life Sciences 1559: 277-286.

9. Holman ER, Vliegen HW, van der Geest RJ, Reiber JHC, van Dijkman PR, et al. (1995) Quantitative analysis of regional left ventricular function after myocardia infarction in the pig assessed with cine magnetic resonance imaging. Magnetic Resonance in Medicine 34: 161-169.

10. Sasayama S, Franklin D, Ross Jr J, Kemper WS, McKown D (1976) Dynamic changes in left ventricular wall thickness and their use in analyzing cardiac function in the conscious dog: A study based on a modified ultrasonic technique. The American Journal of Cardiology 38: 870-879.

11. Sheehan FH, Bolson EL, Dodge HT, Mathey DG, Schofer J, et al. (1986) Advantages and applications of the centerline method for characterizing regional ventricular function.Circulation 74: 293-305.

12. Azhari H, Sideman S, Weiss JL, Shapiro EP, Weisfeldt ML, et al. (1990) Threedimensional mapping of acute ischemic regions using MRI: wall thickening versus motion analysis.Am J Physiol 259: H1492-1503.

13. Pujadas S, Reddy GP, Weber O, Lee JJ, Higgins CB (2004) MR imaging assessment of cardiac function.J MagnReson Imaging 19: 789-799.

14. von Land D, Rao SR, Reiber JHC (1990) Development of an improved centerline wall motion model. IEEE Proceedings of Computers in Cardiology 687-690.

15. van der Geest RJ, de Roos A, van der Wall E E, Reiber JHC (1997) Quantitative analysis of cardiovascular MR images. Int J Card Imaging 13: 247-258.

16. Beohar N, Flaherty JD, Davidson CJ, Vidovich MI, Brodsky A, et al. (2007) Quantitative assessment of regional left ventricular function with cardiac MRI 
Citation: Sliman H, Elnakib A, Beache GM, Elmaghraby A, El-Baz A (2014) Assessment of Myocardial Function from Cine Cardiac MRI Using a Novel 4D Tracking Approach. J Comput Sci Syst Biol 7: 169-173. doi:10.4172/jcsb.1000152

Three dimensional center surface method. Catheterization Cardiovasc. Interv, 69: 5; 721-728.

17. Prasad M, Ramesh A, Kavanagh P, Gerlach J, Germano G, et al. (2009) Myocardial wall thickening from gated Magnetic Resonance images using Laplace's equation.ProcSoc Photo Opt InstrumEng 7260.

18. Khalifa F, Beache GM, Gimel'farb G, Giridharan GA, El-Baz A (2012) Accurate automatic analysis of cardiac cine images.IEEE Trans Biomed Eng 59: 445455

19. Elnakib, Gimel'farb G, Suri JS, El-Baz A (2011) Medical image segmentation: A brief survey, In Multi-Modality State-of-the-Art Medical Image Segmentation and Registration Methodologies 1-39.

20. Khalifa F, El-Baz A, Gimel'farb G, Abo El-Gahr M (2010) Non-invasive imagebased approach for early detection of acute renal rejection. Proceeding of International Conference on Medical Image Computing and Computer-Assisted Intervention 1: 10-18.

21. Elnakib A, Beache GM, Nitzken M, Gimel'farb G, El-Baz A (2011) A new framework for automated identification of pathological tissues in contrast enhanced cardiac magnetic resonance images. Proceeding IEEE International Symposium on Biomedical Imaging 1272-1275

22. Elnakib, Beache GM, Gimel'farb G, El-Baz A (2012) New automated MarkovGibbs random field based framework for myocardial wall viability quantification on agent enhanced cardiac magnetic resonance images. Int $\mathrm{J}$ Cardiovasc Imaging 28: 1683-1698.

23. Khalifa F, Abou El-Ghar M, Abdollahi B, Frieboes HB, El-Diasty T, et al. (2013) A comprehensive non-invasive framework for automated evaluation of acute renal transplant rejection using DCE-MRI.NMR Biomed 26: 1460-1470.
24. Bouman C, Sauer K (1993) A generalized Gaussian image model for edgepreserving MAP estimation.IEEE Trans Image Process 2: 296-310.

25. Besag J (1986) On the statistical analysis of dirty pictures. Journal of the Royal Statistical Society 48: 259-302.

26. El-Baz (2006) Novel stochastic models for medical image analysis.University of Louisville, KY, USA.

27. McDonald JH, John (2009) Handbook of biological statistics. Baltimore, Sparky House Publishing.

28. Maffessanti F, Nesser HJ, Weinert L, Steringer-Mascherbauer R,Niel J, et al (2009) Quantitative evaluation of regional left ventricular function using threedimensional speckle tracking echocardiography in patients with and without heart disease. The American journal of cardiology, 104: 1755-1762.

29. Maron J, Gardin JM, Flack JM, Gidding SS, Kurosaki TT, et al. (1995) Prevalence of hypertrophic cardiomyopathy in a general population of young adults Echocardiographic analysis of 4111 subjects in the CARDIA Study Circulation 92: 785-789.

30. Drazner MH (2011) The progression of hypertensive heart disease. Circulation 123: $327-334$

31. Hassan W, Al-Sergani H, Mourad W, Tabbaa R (2005) Amyloid heart disease. New frontiers and insights in pathophysiology, diagnosis and management.Tex Heart Inst J 32: 178-184.

32. Ross J Jr (1991) Myocardial perfusion-contraction matching. Implications for coronary heart disease and hibernation 83: 1076-1083. 